\title{
Self-Confidence and Anxiety as Intervening Factors in Clinical Decision-Making in Newly Nursing Bachelor Graduates
}

\author{
Beatriz Paulina Espinosa-Rivera*, Laura Morán-Peña, María Aurora García-Piña, \\ Patricia González-Ramírez, Cristina Margarita López-Ruíz
}

National School of Nursing and Obstetrics, National Autonomous University of Mexico, Mexico City, Mexico

Email address:

betypauer@live.com.mx(B.P. Espinosa-Rivera),eneolm@yahoo.com(L. Morán-Peña), augarcia15@hotmail.com (M.A. García-Piña), patriciag07@yahoo.com.mx(P. González-Ramírez),mago7007@gmail.com(C.M. López-Ruíz)

${ }^{*}$ Corresponding author

\section{To cite this article:}

Beatriz Paulina Espinosa-Rivera, Laura Morán-Peña, María Aurora García-Piña, Patricia González-Ramírez, Cristina Margarita López-Ruíz. Self-Confidence and Anxiety as Intervening Factors in Clinical Decision-Making in Newly Nursing Bachelor Graduates. American Journal of Nursing Science. Vol. 8, No. 2, 2019, pp. 59-67. doi: 10.11648/j.ajns.20190802.14

Received: October 22, 2018; Accepted: March 5, 2019; Published: March 27, 2019

\begin{abstract}
The Clinical Decision-Making (CDM) is essential for the nursing professional practice. However, newly nursing bachelor graduates are not always ready to make clinical decisions. Therefore, the identification of self-confidence and anxiety as emotional barriers during this process will allow teaching/learning strategies to generate educational strategies of teachinglearning that support its development. The objective is to evaluate the levels of self-confidence and anxiety in newly nursing bachelor graduates and compare them to each CDM's dimension. The method for this study was descriptive, comparative and cross-sectional. The "Nursing Anxiety and Self-Confidence with Clinical Decision Making Scale (NASC-CDM)" was applied. Results: It was found that $69 \%$ of the newly nursing bachelor graduates had a high level of self-confidence and $66 \%$ a low level of anxiety. Significant statistical differences were found in the levels of self-confidence of the newly nursing bachelor graduates in accordance with their employment situation; the averages in the anxiety level were higher, although there were no significant statistical differences. It can be concluded that the higher level of self-confidence possessed by the newly nursing bachelor graduates, the lower the level of anxiety, with the exception of those who have nursing working experience. It is necessary for nursing educators to emphasize the development of skills for knowing and acting, because students need to incorporate them cognitively and intuitively in the complete clinical scenario, in order to have one or more options of decision. The low self-confidence level and the high anxiety level are emotional barriers that intervene in CDM, because they block or interfere the assertive decision-making process. This explains why deliberative strategies have to be implemented in the curricular level.
\end{abstract}

Keywords: Clinical Decision-Making, Newly Nursing Graduates, Self-Confidence, Anxiety

\section{Introduction}

To carry out the Clinical Decision-Making (CDM) during the professional exercise, nurses in service must be aware of different options for actions once the problems and/or needs have been identified and the cues within each situation have been properly assessed. Thus, a solution can be given through the selection of the best alternatives, by applying the nurse's knowledge, attitudes and cognitive abilities during the nursing process, as well as the use of scientific evidence, collaborative work and exchange of clinical expertise within the members of the interprofessional team.

Several authors have defined CDM as a dynamic, comprehensive and complex process which depends on the volitional act [1]; CDM requires critical and reflexive thinking skills, clinical reasoning to process data and formulate hypothesis $[2,3]$, considering the relevant previous knowledge, both theoretical and practical [4] that allows the 
selection of a choice between the diverse alternatives of action, in order to make clinical judgments which bring as an outcome the assertive care of the patient and at the same time, the solution to health problems [5-7].

However, Gonzalez and cols. [1], White [8] and Wang and cols. [4] observed that it is not common to find fully developed professional competencies in nursing graduates. Such competencies would allow graduates to provide a high quality care or by means of clinical reasoning, make assertive decisions that contribute to provide a sustained care.

Likewise, White [8] pointed out that CDM is a skill that marks a difference between the nursing professional staff and the technical staff due to academic training, since in the higher level, tools for the construction of clinical thinking are acquired.

In the specific case of Mexico, in the undergraduate training aiming at the development of CDM skills, the factors that may represent emotional barriers and affect the process have not been sufficiently studied. Such is the case of selfconfidence, defined as the belief and the feeling of confidence in oneself about the abilities and skills possessed to organize and execute the sources of action required to reach objectives, which involves self-steem and being careful about emotions [8-10].

On the other hand, anxiety is defined as a state of anticipated apprehension and an association of feelings in situations that generate fear, due to the possibility that a negative event may occur, also taking in consideration the self-perception about the skills possessed $[8,11]$.

Since the positive outcomes for a patient depend on the nurse's skills to execute CDM, it is imperative that nursing students begin to develop such skills, thus generating a feeling of safety and reducing anxiety, while carrying out CDM during their supervised educational experiences [8].

It should be noted that in the particular case of our concern, the participants in the study were newly graduates who have recently concluded their studies, therefore lacking the clinical expertise of a nurse already inserted in the work field. In this context, emotional barriers could be exacerbated, especially considering that during the graduates' obligatory social service, carried out at the end of their studies, they must face complex and uncertain circumstances involving conflict of values, which is typical of institutions of second and third healthcare level. In addition, the graduate's performance takes place in less controlled environments, with less supervision and a higher possibility of the occurrence of adverse events.

This is the importance of researching the levels of selfconfidence and anxiety of the newly nursing bachelor graduates to carry out the CDM. Itano and Tschikota point out that the more careful nursing teachers are about the formation of the CDM process and the detection of affective states that may influence it, the higher the possibility to form graduates with enhanced self-confidence and less anxiety while making clinical decisions, provided that the outcome is achieved when carried out deliberately, setting aside the assumption that learning is achieved naturally over time. This statement is later sustained by White [8].

\section{Clinical Decision-Making and Some Intervening Factors}

As pointed out before, the current professional nursing practice requires nurses to develop skills that will allow them to fulfill health needs in increasingly diverse complex scenarios, derived among others, from natural disasters, social confrontations, viral mutations, prevalence and increase of chronic-degenerative diseases, to name a few. These scenarios demand nurses to constantly make effective, timely, scientifically supported decisions that involve the individual, in order to provide an holistic care that will result in the maximum benefit for the patient. Therefore, CDM is an essential competence for the professional practice of nursing.

$\mathrm{CDM}$ is a complex process that requires skills of critical and reflexive thinking and clinical reasoning, to formulate hypothesis based on knowledge and practice, in order to select options of decisions [2, 3], this selection includes the reasons to carry out an action, if the decision is unforeseen or planned [12], the available resources, assessment of possible risks and especially take into consideration the patient's opinion about treatment and care to be provided. Due to its importance, CDM is considered as "the cornerstone of the profession" [8], since the nursing student acquires and develops knowledge, skills and dexterities from an holistic perspective and by means of applying the scientific method. Furthermore, the AACN [13], states that in this academic degree, tools are provided and professional values are generated, which will support and promote CDM, consequently reinforcing the professional identity and autonomy $[12,14-16]$.

For TDC to be successful, there are three supporting factors, according to White [8]: 1)Using resources to gather information and listening fully, 2) Using information to see the big picture, 3) Knowing and acting. Next, each one of them is explained.

1) Using resources to gather information and listening fully: It involves gathering information from the patient or written sources such as the clinical file or laboratory results, the acquisition of verbal and non-verbal cues (gestures or positions) and the assessment of the patient's condition.

2) Using information to see the big picture: Once the cues have been collected, the nurse should interpret which information is relevant or irrelevant to the patient's situation, and if necessary perform another physical assessment in order to fill information gaps and ask specific questions to validate the cues presented.

3) Knowing and acting: This makes reference to incorporating cognitively and intuitively the elements of the complete clinical scenario, in order to have one 
or more options of decision. It should quantify the number of options and determine if it is necessary to seek for assistance, while pondering risks and benefits. In this instance, the decision has to be made based on presented cues, gathered data, synthesis of the clinical scenario, plausible decision options considered and the appraisal of the effectiveness of the chosen decision.

Given the importance of forming increasingly competent nursing professionals to face complex practice problems and scenarios, there is a growing international interest in the development of CDM in the nursing personnel [17]. Therefore, the generation of deliberate cognitive, procedural and attitudinal strategies is imperative for the nursing graduates, so that such strategies can be fully developed. This will ensure that once inserted in the work field, nurses will accurately identify a problem, appraise the cues within a situation, consider the plausible decision options and act in benefit of the patient, with this ability not being "assumed as a learning that is obtained through time" [15].

If nursing professors are more attentive about the formation of the CDM process, students may feel assured in all its steps, through supervised educational experiences. In this sense, White [8] points out the existence of two affective influences called emotional barriers, which have an effect over CDM and an impact on learning: self-confidence and anxiety. The lack of self-confidence and high levels of anxiety can affect the competency in this process.

This is explained by the fact that experiences, in particular those presenting a high clinical risk, make nursing students be alert of the need to think actively about the use of their knowledge in a specific situation [18]. Furthermore, students are constantly in close contact with the patients, thus being the first to observe any change or deterioration in the patient's condition, unlike other members of the multidisciplinary health team. So, nurses should be motivated to apply the CDM process [8] considering all the elements involved in the decision and risks and benefits that any of their interventions may have in the patient's care.

Nursing care is expected to be holistic. Therefore, students' training should match this focus, through self-awareness of their affective states. This will allow students to understand and identify the implication of holism in the care and to raise congruence between what was received and what is expected from them.

\subsection{Self-confidence in CDM}

Self-confidence is the belief and the feeling of confidence regarding oneself, and about self-respect, abilities and skills to organize and execute actions required to reach objectives while foreseeing the effort, perseverance and attitude to overcome obstacles and failures. This involves both selfesteem and being careful about emotions [8, 9]. Selfconfidence encompasses the feelings of well-being that come as a result of the agreement made with oneself and with the surrounding people, while experiencing deep positive emotions that will enable nurses to act courageously and foster self-assessment [10].
For better understanding, self-confidence has been classified in inner and external confidence. The former is related with love for oneself, self-knowledge and positive thinking. The latter is related with communication and control over emotions. These features allow self-confidence to be identified also as self-efficacy $[10,19]$.

Self-confidence is fundamental for the nurse, in order to feel assured while executing an action. Nevertheless, its development depends on repeated experiences that lead to reinforce the perception about success and failure [19]. Moreover, it has been established that self-confidence is also multifactorial, since it is related to cognitive and behavioral interaction, as well as influences from the environment [9]. An environment in which the other members of the multidisciplinary team encourage nurses about CDM, is highly motivating and strengthens the nurses' self-confidence [4].

In the cognitive field, self-confidence regulates human functioning, as it fosters harboring high expectations, visualizing the future, taking challenges and guiding actions, visualizing positive outcomes when focusing on the person's own capacities, and not on deficiencies or on anything that could be deemed as negative [19].

In the case of baccalaureate nursing students, academic performance may be affected by low levels of selfconfidence, due to the influence that the practice of care and teaching strategies have on its development [10]. For this, it is indispensable to reinforce self-confidence during the student's formation. It is necessary for a student to develop leadership skills, achieve a transcendence in the professional behavior, have an optimum academic performance, obtain and increase the clinical competence and mainly, so selfregulation of emotions generated in diverse circumstances can be achieved, while mediating between personal and professional values during CDM, in order to solve problems and provide effective care to patients [14].

Although a high level of self-confidence does not guarantee that a student will maintain a positive attitude in challenging conditions, it will certainly provide cognitive and emotional tools to face complex situations [10] and achieve the planned goals. Accordingly, it has been observed that students who lack self-confidence are prone to falling into risky situations. Conversely, those with a high level in this affective influence are able to maintain control under adverse situations, even reaching the point of relaxing themselves. They are usually, a support for themselves and others, thus achieving a successful CDM process [19].

Higher self-confidence in nursing students will come as a result of constant testing of their knowledge and capacity to select assertive interventions to be executed on patients. Once proven successful, the level of self-confidence will increase, reinforcing every time the assurance about their clinical performance, while reducing the possibility of an error [19]. In the case that students have previous working experience, selfconfidence is enhanced as the student perceives himself as a competent professional to treat people [9].

However, the fact that students' training normally occurs in the clinical scenario, prevents self-confidence to develop 
adequately, since the actions taken by students are usually carried out while presenting high levels of anxiety [20]. Because of this, nursing teachers should be aware of the students' emotions, so that negative outcomes and demotivation can be avoided.

In order that affectations in the students' self-confidence can be prevented, it has been suggested that clinical practices may begin under supervision, to provide assurance. After this, practices have to be without supervision, allowing students to act autonomously and in doing so, stimulate constructive self-criticism [20], encourage increasing self-confidence and maintain it at high levels, to endure and reduce anxiety.

\subsection{Anxiety in CDM}

One of the factors involved in CDM is anxiety, defined as the state of uncertainty and anguish regarding future actions, having in mind the possibility that a negative event may occur. Anxiety generates anticipatory apprehension or intense fear; it is an association of negative feelings in adverse situations, taking into consideration the perception about the acquired skills, triggering a physiological response and an emotional state by propitiating subjective feelings of agitation [8, 11].

Although anxiety and stress are very similar emotions, since both cause a high physiological activation, they can differ because the first is an emotional reaction of alertness in the face of a threat, while the second is a broad process of adaptation to the environment, triggered by the imbalance between the demands of the situation and the resources of the person [21].

Anxiety has repercussions over the human body. As a response to this state, the pressure of carbon dioxide levels triggers suffocation. The anxious person suffers from dyspnea, caused by low activity in the breathing muscles, due to the reduction of activity in the inspiratory muscles and in lung efficiency. The increase in frequency and depth to eliminate the excess of carbon dioxide, makes breathing to be forced and superficial. The autonomic nervous system increases pulse to conduct the blood volume to the digestive organs and the large skeletal muscles, leaving the periphery without sufficient irrigation, thus decreasing the temperature of the skin. As a consequence, epinephrine and norepinephrine are released, to prepare the body for attacking or escaping [22]. These changes alter the fine movements required to carry out clinical actions.

Accordingly, the newly nursing bachelor graduates are subjected to pressure, conflicts and continuous changes which produce high tension that results in higher anxiety, both in their academic formation and professional practice, by demanding the integration of knowledge, technical skills, development of appropriate attitudes and emotional responses to the clinical scenarios, which require a responsible control of clinical situations, to accomplish an efficient and effective performance involved in nursing interventions [21, 22].

In other words, anxiety is present frequently in nurses, because their professional activity involves the offer of direct human services and this causes a process of disturbance by having to mobilize their energy excessively to adapt to working demands and the degree of freedom in making possible decisions, in order to face situations which require quickness and assertiveness [21].

The presence of anxiety affects quality in the healthcare process, triggering absenteeism, antagonism and job dissatisfaction. When these are combined with interpersonal conflicts, the poor social support from co-workers or a lack of rewards and acknowledgments, it poses a major risk to physical and mental health, also causing distress and low motivation in the nursing staff [21].

In the academic field, nursing faculties are governed by programs that demand high standards, expected to be met by students through the assertive percentages in each exam taken. This situation generates anxiety in students, because when tested under these standards, they are liable to feelings of weakness and inability to demonstrate their knowledge and potential, which may translate into poor academic performance [22].

Another factor that favors the appearance of anxiety in nursing students during the clinical practices, lies in the stressful situations where both anguish and satisfaction are present, generated by situations such as death and agony of the patients, conflicts with members of the multidisciplinary team, not adequate preparation for the current clinical demand, lack of support from their mentors and a heavy workload [23].

On account of this, it is of the utmost importance that teachers know and spread coping styles through psychoeducational programs, so that "students who are vulnerable to generate anxiety, can decrease the frequency or avoid the development of coping skills that entail a negative impact" [23].

Self-confidence and anxiety are intrinsic factors that intervene in the teaching and the learning of CDM and it is important to study them, so students acquire educational experiences inside a safe and supervised environment where real situations are simulated, so once their studies are concluded, dexterities will have been developed to face and control these barriers in a better way. Because of this, the aim of the present study was to identify the levels of self-confidence and anxiety in the newly nursing bachelor graduates during the CDM process and to detect if there were differences between graduates, the level of care where they did their social service, and their employment situation.

\section{Methods}

\subsection{Participants}

The participants in the study were 437 newly nursing bachelor graduates of a public university in Mexico City. To calculate the size of the sample at convenience of the formula of finite populations was applied, the total of the sample was 162 participants. Sixteen hospitals were randomly chosen in 
which the graduates were performing their social service. Social service is mandatory in Mexico and during this period, graduates render free professional services to society for a year, as to repay the received education. The inclusion criteria for this study were: nursing graduates had to be performing their social service for the first time, having completed a period of six months; they had to be performing clinical activities at the time of application and finally, they had to agree to participating in the study. Regarding the hospitals, four belonged to the second level of care and twelve hospitals to the third level.

\subsection{Design}

This study was descriptive, comparative and correlational. Self-confidence and anxiety levels of the newly nursing graduates were measured without manipulating the variables. The data collection was done in a single moment. Population groups were contrasted according to the level of attention in which they performed their social service, as well as whether they were working at the time or not.

\subsection{Tools}

The tool "Nursing Anxiety and Self-Confidence with Clinical Decision Making Scale (NASC-CDM)" was applied with previous consent from the author [8]. It measures the levels of self-confidence and anxiety during the CDM process through two subscales, which both form a hybrid scale. It is considered a hybrid scale because it examines the cognitive and intuitive process of making a clinical decision [8]. In the application made by the author, the selfconfidence scale had a Cronbach's Alpha of $\alpha=.97$ and the anxiety scale $\alpha=.96$. The tool evaluates three dimensions: 1 ) using resources to gather information and listening fully, 2) knowing and acting and 3) using information to see the big picture. The tool consists of 27 items and the answers are in a Likert scale with six response options from zero to five: Nothing ( 0 points) to Totally ( 5 points) and the middle option was Something (3 points).

Once the author's consent was obtained for application, the instrument was translated into Spanish and subsequently submitted for back translation to corroborate that the original purpose of the instrument was maintained. For purposes of greater comprehension of the participants, the items of each subscale were separated, leaving a total of 54 items. In this investigation, the reliability of the instrument was $\alpha=.93$.

\subsection{Ethics}

Authorization to conduct the research was requested to the National School of Nursing and Obstetrics (ENEO), which belongs to the National Autonomous University of Mexico (UNAM) and to the hospital managers in which the graduates were performing their social service. The written consent of the graduates to participate in the study was obtained.

\subsection{Data Collection}

A self-administered questionnaire was applied; the graduates had thirty minutes to complete it. A classroom in the hospital was set, in which the graduates were comfortable and calm to solve the instrument. At the end, the applicator verified the complete filling of the tool.

\subsection{Data Analysis}

For the descriptive data, measures of central tendency and dispersion were used. For the comparison between the population groups, the Student's $\mathrm{T}$ test was used for independent samples. The statistical processing was carried out with the SPSS program v. 21.

\section{Results}

About the characterization of the sample, $79 \%$ of the participants were female and $21 \%$, male. The majority of the graduates were in the range of 21-27 years of age; of the total of participants in the study, almost a third part worked and a half of them worked in a nursing-related area (Table 1).

Table 1. Characterization of the sample.

\begin{tabular}{lll}
\hline Characteristics & Frequency & Percentage \\
\hline Sex & & \\
Female & 128 & $79 \%$ \\
Male & 34 & $21 \%$ \\
Age & & \\
$21-27$ years old & 148 & $91 \%$ \\
$28-33$ years old & 11 & $7 \%$ \\
$34-39$ years old & 3 & $2 \%$ \\
School shift attended & & \\
Morning & 96 & $59 \%$ \\
Evening & 55 & $34 \%$ \\
Both & 11 & $7 \%$ \\
Employment situation & & \\
Do not work & 112 & $69 \%$ \\
Work & 50 & $31 \%$ \\
Employment is related to nursing $(\mathrm{n}=50)$ & & \\
Yes & 25 & $50 \%$ \\
No & 25 & $50 \%$ \\
\hline
\end{tabular}

Regarding the levels of self-confidence and anxiety of the newly nursing bachelor graduates, it was found that $69 \%$ had a high level of self-confidence and $66 \%$ a low level of anxiety (Figure 1).

An analysis of outcomes was made for each of the three dimensions of the CDM process and it was found that $68 \%$ of the newly nursing bachelor graduates had a high level in the dimension Using resources to gather information and listening fully, and also had a high level of self-confidence (table 2). In the case of those who had the higher level in Using information to see the big picture, $61 \%$ also had a high level of self-confidence, and those who demonstrated a low level on this dimension, did not have medium or high levels of self-confidence (table 2). Finally, in the dimension Knowing and acting, a little more than half of the newly nursing bachelor graduates had a high level and also had a high level in self-confidence (table 2). 


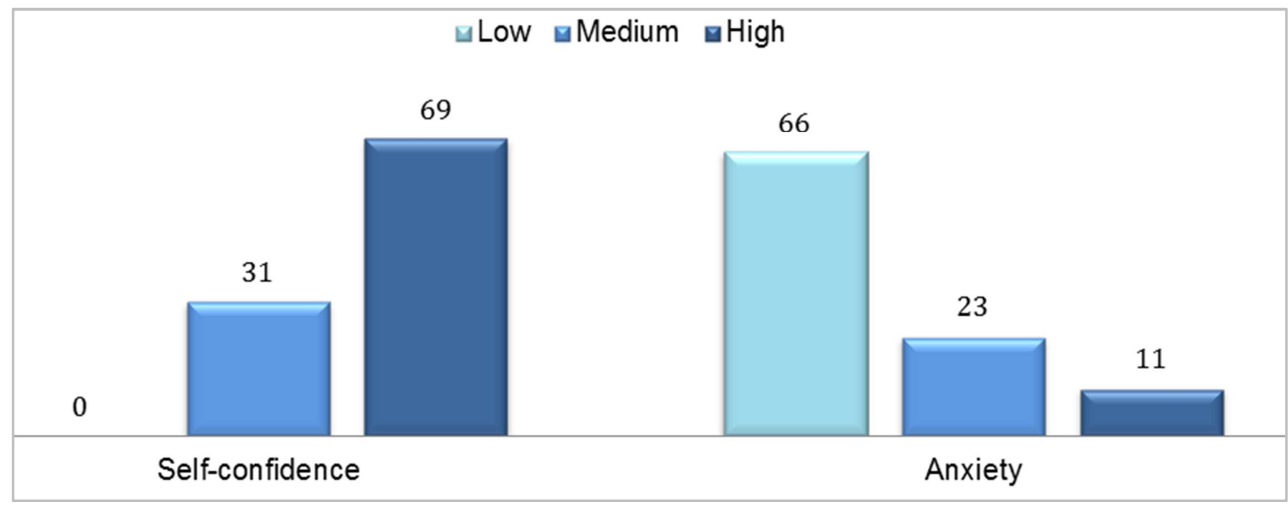

Figure 1. Levels of self-confidence and anxiety of the newly nursing bachelor graduates (\%).

Table 2. Levels of the dimensions of the NASC-CDM in comparison to the levels of Self-confidence.

\begin{tabular}{llll}
\hline Levels obtained by the newly nursing bachelor graduates & & & \\
\hline \multirow{2}{*}{ Dimension } & Self-confidence $\mathbf{n}=\mathbf{1 6 2}$ & & High \\
\cline { 2 - 4 } & Low & Medium & Fo. (\%) \\
\hline Using resources to gather information and listening fully & Fo. (\%) & Fo. (\%) & 0 \\
Low & 0 & $2(1)$ & $2(1)$ \\
Medium & 0 & $36(22)$ & $109(68)$ \\
High & 0 & $13(8)$ & 0 \\
Using information to see the big picture & & $2(1)$ & $13(8)$ \\
Low & 0 & $46(28)$ & $98(61)$ \\
Medium & 0 & $3(2)$ & 0 \\
High & 0 & & $2(1)$ \\
Knowing and acting & & $46(28)$ & $88(55)$ \\
Low & 0 & $3(2)$ & \\
Medium & 0 & 0 & \\
\hline
\end{tabular}

In the case of the levels of Using resources to gather information and listening fully contrasted with anxiety levels, just $10 \%$ of the newly nursing bachelor graduates that had a high level in this dimension also had a high level of anxiety. In the high level of Using information to see the big picture, $10 \%$ of the newly nursing bachelor graduates had a high level of anxiety, 2\% a medium level and none presented a low level. About the high level of Knowing and acting, 10\% of the newly nursing bachelor graduates had a high level of anxiety and no one demonstrated a low level contrasted with the level of this dimension (table 3 ).

Table 3. Levels of the dimensions of the NASC-CDM in comparison to the levels of Anxiety.

\begin{tabular}{llll}
\hline Levels obtained by the newly nursing bachelor graduates & & & \\
\hline \multirow{2}{*}{ Dimension } & Anxiety $\mathbf{n = 1 6 2}$ & Medium & High \\
\cline { 2 - 4 } & Low & Fo. (\%) & Fo. (\%) \\
\hline Using resources to gather information and listening fully & Fo. $(\%)$ & $7(4)$ & 0 \\
Low & $104(64)$ & $30(19)$ & $1(1)$ \\
Medium & $3(2)$ & 0 & $17(10)$ \\
High & 0 & $2(1)$ & 0 \\
Using information to see the big picture & & $32(20)$ & $2(1)$ \\
Low & $94(58)$ & $3(2)$ & $16(10)$ \\
Medium & $13(8)$ & & \\
High & 0 & $4(3)$ & 0 \\
Knowing and acting & & $31(19)$ & $2(1)$ \\
Low & $88(54)$ & $2(1)$ & $16(10)$ \\
Medium & $19(12)$ & 0 & \\
High & & & \\
\hline
\end{tabular}

In regard to statistical significant differences in the levels of self-confidence and anxiety of the graduates according to the level of attention in which their social service was being performed, there were no differences between those performing it in the 
second level of attention and those in the third level $(t=-1.323$, $g l=160, p=.188)$ and $(t=.702, g l=160, p=.484)$, respectively.

About self-confidence of the newly nursing bachelor graduates according to their employment situation, there were statistical significant differences between the group that worked and the one who did not $(t=-2.409, g l=160, p=.017)$. The group that worked, obtained a higher average (106.59) compared to the group who did not work (97.91) (Figure 2).

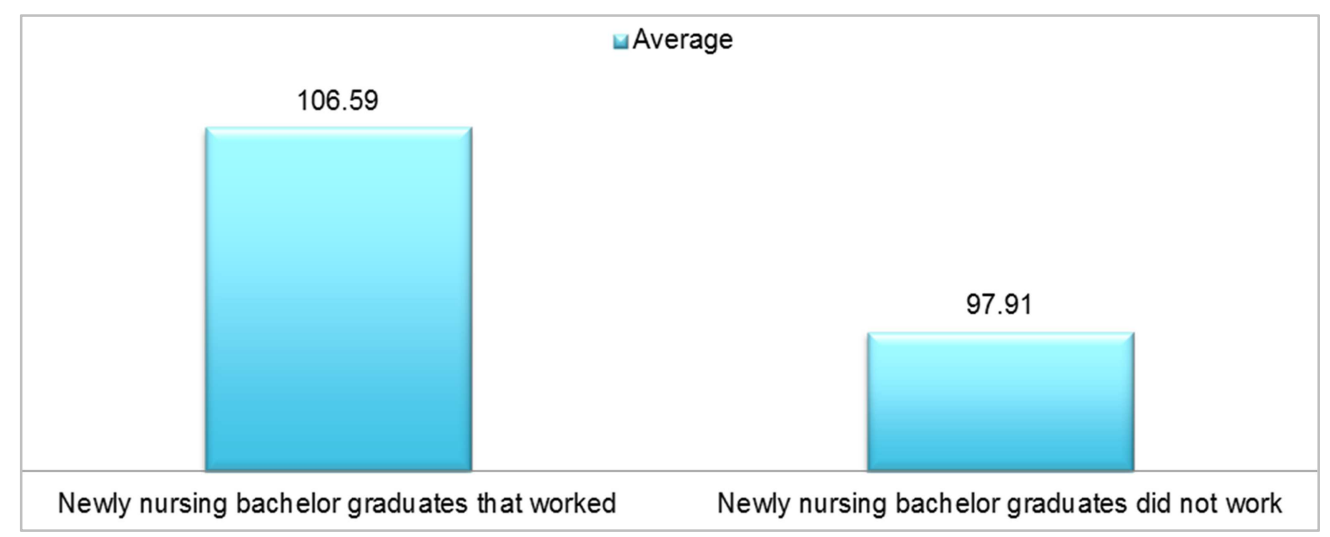

Figure 2. Average of self-confidence of the newly nursing bachelor graduates according their employment situation (Max. possible 135) ( $t=-2.409$, gl=160, $p=.017$ ).

Regarding the anxiety of the newly nursing bachelor graduates according to their employment situation, even those who worked obtained a higher average (43) compared to those who did not (39.75); there were no statistical significant differences $(t=.588, g l=160, p=.557)$.

\section{Discussion}

Due to the fact that the newly nursing bachelor graduates are practitioners living a professional transition process [2426], they would be expected to present low levels of selfconfidence and a high level of anxiety, especially those who were in a third-level institution. Nevertheless, in this study as in White's [8], it was not the case.

The resources of information and listening fully are essential in newly nursing bachelor graduates, because these resources can strengthen communication and therefore maintain self-confidence, a necessary element for starting and developing the CDM process. This may be explained because the newly nursing bachelor graduates are newcomers who need to support their clinical decisions in rules and in the information received in their academic training -as pointed out by Benner-, for this reason the higher the level of use of resources to gather information and listening fully, the greater the self-confidence $[8,16]$.

In this study as in White's(8), the newly nursing bachelor graduates with a high level of self-confidence use the information to perceive all the elements of the general view, which allow them to elaborate different decision options and execute the necessary interventions. That is to say, nurses who perceive themselves as professionals, have the skill to integrate all the elements in the general view.

Allegedly, when the newly nursing bachelor graduates possess knowledge, they build up their self-confidence, and the feeling of self-competency enables them to act in a safe way in the CDM process [19] decreasing both anxiety and the possibility of making mistakes.
Graduates who had a low level in the Using resources to gather information and listening fully dimensions, showed that they were themselves a little anxious in this situation, while the newly nursing bachelor graduates who had a high level in this dimension, were more anxious. This contradicts White's statement [8] about newly nursing bachelor graduates having a high level of Using resources to gather information and listening fully, and a low level of anxiety.

This study found that newly nursing bachelor graduates who had a high level in the dimension Knowing and acting, also had a high level of anxiety; this could reinforce the idea that novice nurses often need to integrate knowledge, technical skills, develop and increase the CDM skills and through this trajectory, generate high levels of anxiety as stated in the research by Flores-Villavicencio and cols. [21]

This research agrees with White [8] about a high level of self-confidence being auspicious to high levels in the dimensions of the CDM with low levels of anxiety, so the importance of these factors in the process is confirmed.

According to the outcomes of this study, the newly nursing bachelor graduates that worked had a high level of selfconfidence, which is congruent with the study by Sergeev and cols. [20] about self-confidence increasing when the graduates also work; although the authors measured the level of self-confidence in simulated practices with mannequins, it could be equivalent in our newly nursing bachelor graduates to the reinforcement experimented when, besides doing their social service, they work.

\section{Conclusions}

It is concluded that a high level of self-confidence in the newly nursing bachelor graduates contributes to regulate their anxiety level during the CDM process, as the obtained results illustrate. This is liable to be reflected in the professional exercise and derive in safe, responsible and quality care practices. 
The emotional barriers increase or decrease the success of the CDM process. Therefore, an important task during the professional training is to propitiate the acquisition of knowledge and skills for the development of self-confidence and the reduction of anxiety.

The high level of self-confidence is directly proportional to that of the three dimensions of CDM, as it indicates that the newly nursing graduates provide timely and accurate attention; this surely will start new research projects that deepen in how this emotional barrier has repercussions and impacts on CDM.

The newly nursing bachelor graduates are considered newcomers who adhere to established rules and procedures, which can favor the systematization of care and affect the application of the nursing care process by avoiding or minimizing risks to the person and providing a quality care.

The newly nursing bachelor graduates with a high level in the dimension of knowing and acting, feel themselves not necessarily safe in this dimension, which is reflected in their initiative and their timely acting at the moment of making clinical decisions.

The level of anxiety of the newly nursing bachelor graduates is proportional to the level of using resources to gather information and listening fully. The lack of knowledge would be expected to generate a high level of anxiety; however, it was possible to notice through this study that low levels of anxiety reflect disinterest about not knowing. Nevertheless, it is also a mental process in which the newly nursing bachelor graduates reflect on the decision to be made.

Novice nurses present difficulties to integrate all the elements of the big picture in their development process; this requires the implementation of learning strategies in the classroom, laboratory and in the clinical practice scenario, to complete the CDM process that enhances the level of selfconfidence.

The last two steps of the CDM process involve the possession of knowledge and the ability to know how, where and when to apply it; therefore it is a competency expected to have been developed at the time of graduation. In contrast, more than a half of the newly nursing bachelor graduates obtained a low level in this dimension and low anxiety, which leads to think they probably consider it a competency acquired over time and not worthy of generating anxiety. All of the above suggests the modification of traditional teaching inside the classroom, through interactive sessions with case studies, problem-based learning and the use of technology for care.

Finally it should be noted that the newly nursing bachelor graduates that work and simultaneously do their social service, have a high level of self-confidence which would result in assuming greater responsibility in the CDM and reducing risks and omissions in the care. Similarly, they can generate a high level of anxiety, due to their greater professional experience.

\section{Implications for the Practice and the Education in Nursing}

Teacher observation of students, allows to detect the occurrence and/or development of self-confidence and anxiety, which makes it possible to intervene in a timely manner to prevent negative consequences. This guarantees that the care to be provided will be effective when making appropriate clinical decisions.

Due to the internationally existing demand for nursing education to teach and use educational theories and models for the CDM, its implementation is necessary through teaching strategies of this process within the curricular plan. In such a way, graduates will be guided professionally in the CDM by being stimulated through their critical thinking and their clinical reasoning.

It has been established that nursing education must have a holistic approach [16], so if nursing teachers direct their attention not only to the building of knowledge but also to recognizing the students' minds, bodies and emotions, they may reinforce their self-confidence and in the case of anxiety, make it decrease.

Major experience in the clinical practice contributes to the development of skills and competencies, increasing selfconfidence and contributing to decrease and control anxiety, hence the importance of the teaching laboratory practices with clinical simulation that foster situations similar to those in reality, inside a safe and supervised environment, which contributes to the professional exercise.

Regarding the curricular plan, it is essential to incorporate deliberate strategies for the development of critical thinking, clinical reasoning and CDM.

\section{Acknowledgements}

To the General Office of Academic Personnel Affairs of the National Autonomous University of Mexico, for financing through project IN302614 Resolution of problems and clinical decision making in nursing graduates. The perspective of reflective practice.

\section{References}

[1] González Velázquez MS, Morán Peña L, Sotomayor Sánchez S, León Moreno Z, Espinosa Olivares A, Paredes Breña L. A comparative study of the ways of making decisions in recently enrolled and advanced nursing students. Perfiles educativos. 2011; 33:134-43.

[2] Jefford E, Fahy K, Sundin D. Decision-Making Theories and their usefulness to the midwifery profession both in terms of midwifery practice and the education of midwives. International Journal of Nursing Practice. 2011; 17(3):24653.

[3] Roche JP. A Pilot Study of Teaching Clinical Decision Making With the Clinical Educator Model. J Nurs Educ. 2002; 41(8):365-7. 
[4] Wang Y, Chien W-T, Twinn S. An exploratory study on baccalaureate-prepared nurses' perceptions regarding clinical decision-making in mainland China2011. 1706-15 p.

[5] Aguilera-Rivera M, Morán-Peña L, Espinosa-Rivera BP, Galicia-Luna A， López-Ruíz CM， García-Piña MA. Experiences by nursing graduates in clinical decision-making. Rev. iberoam. Educ. investi. Enferm. 2017; 7(3):9-18.

[6] Morán-Peña L， García-Piña MA， González-Ramírez P, Godínez-Bueno SY, Aguilera-Rivera M. Problem-solving and decision-making, essence of reflexive practice in nursing: A literature review. Enfermería Universitaria. 2016; 13(1):47-54.

[7] Cameron ME, Schaffer M, Park H-A. Nursing students experience of ethical problems and use of ethical decisionmaking models. Nursing Ethics. 2001; 8(5):432-47.

[8] White KA. The Development and Validation of a Tool to Measure Self-Confidence and Anxiety in nursing Students While Making Clinical Decisions. Las Vegas, Nevada: University of Nevada; 2011.

[9] Chesser-Smyth PA, Long T. Understanding the influences on self-confidence among first-year undergraduate nursing students in Ireland. Journal of Advanced Nursing. 2013; 69(1):145-57.

[10] Kukulu K, Korukcu O, Ozdemir Y, Bezci A, Calik C. Selfconfidence, gender and academic achievement of undergraduate nursing students. Journal of Psychiatric and Mental Health Nursing. 2013; 20:330-35.

[11] Chernomas WM, Shapiro C. Stress, Depression, and Anxiety among Undergraduate Nursing Students. International Journal of Nursing Education Scholarship. 2013; 10(1):255-66.

[12] Standing M. Clinical judgement and decision making for nursing students. 2a ed. Standing SBaM, editor. London: SAGE; 2014

[13] ACCN. The Essentials of Baccalaureate Education for Professional Nursing Practice Whashington: American Association of Colleges of Nursing; 2008 [cited 2016 October 26]. Available from: http://www.aacn.nche.edu/educationresources/BaccEssentials08.pdf.

[14] Iacobucci TA, Daly BJ, Lindell D, Griffin MQ. Professional values, self-esteem, and ethical confidence of baccalaureate nursing students. Nursing Ethics. 2012; 20(4):479-90.
[15] Baxter P, Boblin S. Decision Making by Baccalaureate Nursing Students in the Clinical Setting. J Nurs Educ. 2008; 47(8):345-50.

[16] Tadeu-Shinyashiki G, Costa-Mendes IA, Trevizan MA, Day RA. Professional socialization: students becoming nurses. Rev Latino-am Enfermagem. 2006; 14(4):1-10.

[17] Darbyshire C, Fleming VEM. Governmentality, student autonomy and nurse education. Journal of Advanced Nursing. 2008; 62(2):172-9.

[18] Benner P, Sutphen M, Leonard V, Day L. Educating nurses. A call for radical transformation. Vista Lane, Stanford, California: Jossey-Bass; 2010.

[19] Amado-Martins JC, Negrão-Baptista RC, Dias-Coutinho VR, Mazzo A, Alves-Rodrigues, Costa-Mendes IA. Selfconfidence for emergency intervention: adaptation and cultural validation of the Self-confidence Scale in nursing students. Rev Latino-am Enfermagem. 2014:1-8.

[20] Sergeev I, Lipsky AM, Ganor O, Lending G, Abebe-Campino MG, Morose MA, et al. Training modalities and SelfConfidence building in performance of life-saving procedures. Military Medicine. 2012; 177(8):901-06.

[21] Flores-Villavicencio ME, Troyo-Sanromán R, Valle-Barbosa MA, Vega-López MG. Anxiety and stress in nursing staff practice in a third-level hospital in Guadalajara. Revista Electrónica de Psicología Iztacala. 2010; 13(1):1-17.

[22] Prato CA, Yucha CB. Biofeedback-assisted relaxation training to decrease test anxiety in nursing students. Nurs Educ Perspect. 2013; 34(2):76-81.

[23] Shikai N, Shono M, Kitamura T. Effects of coping styles and stressful life events on depression and anxiety in Japanese nursing students: A longitudinal study. International Journal of Nursing Practice. 2009; 15(3):198-204.

[24] Raile-Alligood M, Marriner-Tomey A. Modelos y teorías en enfermería. 7 ed. Barcelona: ELSEVIER; 2011.

[25] Meléis AI. Transitions Theory Middle Range and Situation Specific Theories in Nursing Research and Practice. New York: Springer Publishing Company; 2010.

[26] Bandura A. Self-efficacy: the exercise of control. New York: Freeman; 1998. 